\title{
Efficient Reconstruction of RC-Equivalent Strings
}

\author{
Ferdinando Cicalese ${ }^{1}$, Péter L. Erdős ${ }^{2}$, and Zsuzsanna Lipták ${ }^{3}$ \\ 1 Dipartimento di Informatica ed Applicazioni, University of Salerno, Italy \\ cicalese@dia.unisa.it \\ 2 Alfréd Rényi Institute of Mathematics, Budapest, Hungary \\ elp@renyi.hu \\ 3 AG Genominformatik, Technische Fakultät, Bielefeld University, Germany \\ zsuzsa@cebitec.uni-bielefeld.de
}

\begin{abstract}
In the reverse complement (RC) equivalence model, it is not possible to distinguish between a string and its reverse complement. We show that one can still reconstruct a binary string of length $n$, up to reverse complement, using a linear number of subsequence queries of bounded length. A simple information theoretic lower bound proves the number of queries to be tight. Our result is also optimal w.r.t. the bound on the query length given in [Erdős et al., Ann. of Comb. 2006].
\end{abstract}

\section{Introduction}

Reconstructing a string over a finite alphabet $\Sigma$ from information about its subsequences is a classic string problem, with applications ranging from coding theory to bioinformatics. Because of the confusion in terminology in the literature, we want to give a precise defintion right here: Given two strings s, $\mathbf{t}$ over $\Sigma$, $\mathbf{s}=s_{1} \ldots s_{n}$ and $\mathbf{t}=t_{1} \ldots t_{m}$, we say that $\mathbf{t}$ is a subsequence (often called subword) of $\mathbf{s}$ if there exist $1 \leq i_{1}<i_{2}<\ldots<i_{m} \leq n$ such that $\mathbf{t}=s_{i_{1}} s_{i_{2}} \ldots s_{i_{m}}$. It was shown by Simon in 1975 [12] that two strings of length $n$ are equal if their subsequences up to length $\lfloor n / 2\rfloor+1$ coincide. The proof, as given in Chapter 6 of the classic Lothaire book [11] can be easily adapted to yield an algorithm which reconstructs the string $\mathbf{s}$ of length $n$, using $O(|\Sigma| n)$ queries of the type "Is $\mathbf{u}$ a subsequence of $\mathbf{s}$ ?" Here, $\mathbf{u}$ is a string of length at most $\lfloor n / 2\rfloor+1$.

In this paper, we consider this problem in the RC-equivalence model, which is motivated by reverse complementation of DNA. Our alphabet consists of pairs of characters $(a, \bar{a})$, called complement pairs, and for every string $\mathbf{s}$ over $\Sigma$, $\mathbf{s}=s_{1} \ldots s_{n}$, we define its reverse complement as $\tilde{\mathbf{s}}=\bar{s}_{n} \ldots \bar{s}_{1}$. Two strings $\mathbf{s}, \mathbf{t}$ are $\mathrm{RC}$-equivalent if $\mathbf{s}=\mathbf{t}$ or $\mathbf{s}=\tilde{\mathbf{t}}$. A string $\mathbf{u}$ is an RC-subsequence of $\mathbf{s}$ if $\mathbf{u}$ or $\tilde{\mathbf{u}}$ is a subsequence of $\mathbf{s}$. Erdôs et al. showed in [4] that two strings $\mathbf{s}$ and $\mathbf{t}$ of length $n$ are equal if all their RC-subsequences up to length $\left\lceil\frac{2}{3}(n+1)\right\rceil$ coincide. However, no reconstruction algorithm was given.

Here we present such an algorithm for the case of a binary alphabet, i.e., where the alphabet consists of two complementary characters. Our algorithm 
reconstructs a string $\mathbf{s}$ of length $n$, using $O(n)$ queries of the type "Is $\mathbf{u}$ an RCsubsequence of $\mathbf{s}$ ?" where $\mathbf{u}$ is a string of length at most $\left\lceil\frac{2}{3}(n+1)\right\rceil$. We note that our algorithm is optimal both w.r.t. the length of the queries, and w.r.t. the information theoretic lower bound on the number of queries necessary for exact reconstruction. We also give a simple algorithm for arbitrary alphabets, adapted from a paper by Skiena and Sundaram [13], where the length of the queries is not bounded, using $O(n \log |\Sigma|)$ queries.

It should be noted that the problem differs considerably from the classical model. For example, consider the string $\mathbf{s}=\bar{a} \bar{b} a \bar{a} b$. Then $a b a$ is not a subsequence of $\mathbf{s}$, but it is an RC-subsequence, because $\bar{a} \bar{b} \bar{a}$ is a subsequence of $\mathbf{s}$.

The RC-equivalence model can be viewed as a special case of erroneous information, where the answers to subsequence queries could be either about the query string or its reverse complement. It is also a special case of a group action on $\Sigma^{*}$, the set of finite strings over $\Sigma$. The search in $\Sigma^{n}$ is substituted by a search in $\Sigma^{n} / \sim$, where $\sim$ is the equivalence induced by the group action.

Related work. Most literature deals with the classical, i.e. non-RC, model. In addition to the papers mentioned above, we want to point to the following.

When the multiset of subsequences is known, then much shorter subsequences suffice to uniquely identify a string: A string of length $n$ can be uniquely identified by the multiset of its subsequences of length $\left\lfloor\frac{16}{7} \sqrt{n}\right\rfloor+5$, as shown by Krasikov and Roditty [6]. Dudík and Schulman [3] give asymptotic lower and upper bounds, in terms of $k$, on the length of strings which can be uniquely determined by the multiset of their subsequences of length $k$.

Levenshtein [7] investigates the maximal number of common subsequences of length $k$ that two distinct subsequences of length $n$ can have. Here, subsequences are regarded as erroneous versions of the original string. The aim is to find how many times a transmission needs to be repeated, over a channel which allows a constant number of deletions, to make unique recovery of the original message possible.

The case where substrings are considered has also received much attention. Substrings, often called factors, are contiguous subsequences: $\mathbf{t}$ is a substring of $\mathbf{s}$ if there are $1 \leq i \leq j \leq n$ such that $\mathbf{t}=s_{i} \ldots s_{j}$. The length of substrings of a string $\mathbf{s}$ of length $n$ which are necessary for uniquely determining $\mathbf{s}$ depends on a parameter of $\mathbf{s}$, namely on the maximal length of a repeated substring, as shown by de Luca and Carpi in a series of papers [2,1]. An algorithm for reconstruction was given by Fici et al. in [5], while the uniqueness bound for multisets of substrings was recently shown to be $\left\lfloor\frac{n}{2}\right\rfloor+1$ by Piña and Uzcágetui [9].

The problem of reconstructing a string of length $n$ using substring queries has also been extensively studied in the setting of Sequencing by Hybridization (SBH), first suggested by Pevzner [8]. Here, a large number of strings of a certain length are queried in parallel, using a DNA chip, and the resulting answers are then used to reconstruct all or parts of the DNA string. A number of different SBH techniques have been proposed, leading to different string combinatorial questions. (See, for example, $[14,10]$ for some more recent results.)

Due to space limitations, some proofs are deferred to the Appendix. 


\section{Preliminaries}

By a paired alphabet we understand a finite set $\Sigma=\left\{a_{1}, \ldots, a_{2 \delta}\right\}$, for some integer $\delta \geq 1$, together with a non-identity involution operation ${ }^{-}: \Sigma \mapsto \Sigma$, which we call complement. Thus, for each $i=1, \ldots, 2 \delta$, there is a $j \neq i$ such that $a_{i}=\overline{a_{j}}$. Notice that by definition, $\overline{\overline{a_{i}}}=a_{i}$, for each $i$.

Let $\mathbf{s}=s_{1} \ldots s_{n}$ be a string (or word) over $\Sigma$, i.e., $\mathbf{s} \in \Sigma^{*}=\bigcup_{i=0}^{\infty} \Sigma^{i}$, where, following standard notation, $\Sigma^{i}=\left\{x_{1} \ldots x_{i} \mid x_{k} \in \Sigma\right.$, for each $\left.k=1, \ldots, i\right\}$,

and $\Sigma^{0}$ is the singleton containing only the empty string $\epsilon$. For each $x \in \Sigma$ we also set $x^{0}=\epsilon$. The reverse complement of $\mathbf{s}$ is defined as $\tilde{\mathbf{s}}=\overline{s_{n}} \overline{s_{n-1}} \ldots \overline{s_{1}}$. Two strings $\mathbf{s}, \mathbf{t}$ are RC-equivalent, denoted $\mathbf{s} \equiv_{\mathrm{RC}} \mathbf{t}$, if either $\mathbf{s}=\mathbf{t}$ or $\mathbf{s}=\tilde{\mathbf{t}}$. For a string $\mathbf{s}=s_{1} \ldots s_{n}$ over the alphabet $\Sigma$, we denote by $|\mathbf{s}|=n$ the length of $\mathbf{s}$, and by $|\mathbf{s}|_{a}=\left|\left\{i \mid s_{i}=a\right\}\right|$ the number of $a$ 's in $\mathbf{s}$, for $a \in \Sigma$.

Given two strings $\mathbf{s}, \mathbf{t}$ over $\Sigma, \mathbf{s}=s_{1} \ldots s_{n}, \mathbf{t}=t_{1} \ldots t_{m}$, we say that $\mathbf{t}$ is a subsequence $^{4}$ of $\mathbf{s}$, denoted by $\mathbf{t} \prec \mathbf{s}$, if there exist $1 \leq i_{1}<i_{2}<\ldots<i_{m} \leq$ $n$ such that $\mathbf{t}=s_{i_{1}} s_{i_{2}} \ldots s_{i_{m}}$. Further, we define $\mathbf{t}$ to be an $R C$-subsequence, denoted $\mathbf{t} \prec_{\mathrm{RC}} \mathbf{s}$ if and only if $\mathbf{t} \prec \mathbf{s}$ or $\mathbf{t} \prec \tilde{\mathbf{s}}$, i.e., if $\mathbf{t}$ is a subsequence of $\mathbf{s}$ or of its reverse complement. Note that the condition $\mathbf{t} \prec \tilde{\mathbf{s}}$ is equivalent to $\tilde{\mathbf{t}} \prec \mathbf{s}$.

Example 1. Our motivating example is the alphabet of the 4 nucleotides (DNA) $\Sigma=\{\mathrm{A}, \mathrm{C}, \mathrm{G}, \mathrm{T}\}$ where $(\mathrm{A}, \mathrm{T})$ and $(\mathrm{G}, \mathrm{C})$ are complement pairs. Let $\mathbf{s}=$ ACCGATTAC. Then $\tilde{\mathbf{s}}=$ GTAATCGGT, GTTT $\nprec \mathbf{s}$ but GTTT $\prec_{\mathrm{RC}} \mathbf{s}$.

We are now ready to state the problem we investigate in the present paper.

The RC-String Identification Problem. Fix a paired alphabet $\Sigma$, together with a string $\mathbf{s}$ over $\Sigma$, and let $n=|\mathbf{s}|$. For any positive integer $T \leq n$, a $T$-bounded RC-subsequence query is any $\mathbf{t} \in \bigcup_{i=1}^{T} \Sigma^{i}$. The answer to such a query is yes (or positive) if and only if $\mathbf{t} \prec_{\mathrm{RC}} \mathbf{s}$. Otherwise the answer is no (or negative). Given the alphabet $\Sigma$, the size of the string $n$, and the threshold on the length of the queries $T \leq n$, the RC-String Identification Problem asks for the minimum number of $T$-bounded RC-subsequence queries which are sufficient to determine the pair $(\mathbf{s}, \tilde{\mathbf{s}})$, for any unknown string $\mathbf{s}$ of size $n$.

We first present an information theoretic lower bound that holds even in the case of unbounded questions, i.e. if $T=n$.

Proposition 2 (Lower Bound). Given a string $\mathbf{s}$ of size $n$ from an alphabet $\Sigma$. Any deterministic algorithm that identifies $\mathbf{s}$ (up to reverse complement) by asking $R C$-subsequence queries needs at least $(n-1) \log |\Sigma|$ queries.

Proof. Upon identifying a string with its reverse complement, there are at most $\left|\Sigma^{n}\right| / 2$ possible distinct strings of length $n$. Any query $\mathbf{t}$ splits the space of candidate solutions into two parts. Therefore, at least $\log \left|\Sigma^{n}\right| / 2=(n-1) \log |\Sigma|$ questions are necessary to identify $\mathbf{s}$.

\footnotetext{
${ }^{4}$ In the literature, the term 'subword' is also common. However, 'subword' is also used to mean a contiguous subsequence. We avoid the term.
} 


\section{Unbounded query size}

If $T=n$ (i.e., no constraint is set on the length of a query), then it is easy to reconstruct a string in linear time. We adapt a simple algorithm from [13], originally developed for the classic case (where queries would answer no if the subsequence only appears in the reverse complement of the string).

Theorem 3. There exists an algorithm for reconstructing a string using $\Theta(n \log |\Sigma|)$ $R C$-subsequence queries of unbounded length.

Proof. (Sketch.) For the binary case $\Sigma=\{a, b\}$, we first find $A:=\max \left\{|s|_{a},|s|_{b}\right\}$ by asking queries $a^{\chi}$ for $\chi=1,2,3 \ldots$ Clearly, $A=\chi-1$ for the first $\chi$ that gives a no answer. Now there are indices $0 \leq i_{0}, i_{1}, \ldots, i_{A}$ s.t. $\mathbf{s}=b^{i_{0}} a b^{i_{1}} a \ldots a b^{i_{A-1}} a b^{i_{A}}$. We find $i_{0}$ by asking $b a^{A}, b^{2} a^{A}, b^{3} a^{A}$, etc., then find $i_{1}$ by asking $b^{i_{0}} a b a^{A-1}, b^{i_{0}} a b^{2} a^{A-1}$ etc. The total number of queries is at most $\frac{3}{2} n+2$.

Now let $\Sigma=\left\{a_{1}, \overline{a_{1}}, \ldots, a_{\delta}, \overline{a_{\delta}}\right\}$. For each complement pair $a_{i}, \bar{a}_{i}$, we first determine $\mathbf{s}_{\mid i}$, the longest subsequence of $\mathbf{s}$ which consists only of $a_{i}$ 's and $\bar{a}_{i}$ 's. This can be done by using the algorithm for the binary case sketched above. Now we iteratively interleave the projections: first $\mathbf{s}_{\mid 1}$ with $\mathbf{s}_{\mid 2}$, yielding $\mathbf{s}_{\mid 1,2}$, then $\mathbf{s}_{\mid 1,2}$ with $\mathbf{s}_{\mid 3,4}$ etc. Interleaving two strings $\mathbf{u}, \mathbf{v}$ that only contain characters from different complement pairs can be done with $2(|\mathbf{u}|+|\mathbf{v}|+1)$ queries using the same idea as for the binary case, with the following small alteration: Since either $\mathbf{u}$ and $\mathbf{v}$, or $\mathbf{u}$ and $\tilde{\mathbf{v}}$ have to be interleaved, we start with $\mathbf{u}$ and $\mathbf{v}$, and if we get a contradictory answer at some point, then we start over with $\mathbf{u}$ and $\tilde{\mathbf{v}}$ (hence the factor 2). So the total number of queries for interleaving the projections $\mathbf{s}_{\mid i}$ is at most $2 n \log \delta+2(\delta-1)$. The number of queries of the first phase is at most $\sum_{i=1}^{\delta}\left(\frac{3}{2} A_{i}+2\right)$, where $A_{i}=|s|_{a_{i}}+|s|_{\bar{a}_{i}}$, yielding $O(n \log |\Sigma|)$ questions in total, using the (natural) assumption that $|\Sigma|=O(n)$.

\section{Bounded query size (binary alphabet)}

We now turn to subsequence queries whose length is bounded by a threshold $T$. In the following, the alphabet is binary, i.e., $\Sigma=\{a, b\}$, with $b=\bar{a}$. The following result shows that string identification by $T$-bounded subsequence queries cannot be attained in general if the threshold $T$ on the size of the subsequence queries is set below $\left\lceil\frac{2}{3} n\right\rceil$. The proof can be found in the Appendix.

Fact 1 (Erdös et al., 2006 [4]) For any $n \geq 4$ there exist two distinct strings of size $n$ with exactly the same set of subsequences of length up to $\left\lceil\frac{2 n}{3}\right\rceil-1$.

This implies that if we are looking for algorithms which are able to reconstruct any binary string of size $n$, we must allow queries of size $\geq\lceil 2 n / 3\rceil$.

Any string $\mathbf{s}$ over $\Sigma$ can be written uniquely in its runlength encoded form:

$$
\mathbf{s}=a^{x_{1}} b^{y_{1}} a^{x_{2}} b^{y_{2}} \ldots a^{x_{\rho-1}} b^{y_{\rho-1}} a^{x_{\rho}} b^{y_{\rho}},
$$


with $x_{1}$ and $y_{\rho}$ possibly 0 , and all other $x_{i}, y_{i}>0$. The number of non-zero $x_{i}, y_{i}$ is the number of runs of $\mathbf{s}$. We denote by $A=|\mathbf{s}|_{a}$ the number of $a$ 's and by $B=|\mathbf{s}|_{b}$ the number of $b$ 's in $\mathbf{s}$. In the following we assume that $A \geq B$. This is without loss of generality since otherwise, swap $\mathbf{s}$ and $\tilde{\mathbf{s}}$. We will denote by $\rho_{a}$ the number of $a$-runs, and by $\rho_{b}$ the number of $b$-runs of $\mathbf{s}$. Note that both have value either $\rho$ or $\rho-1$. (We have $\rho_{a}=\rho_{b}=\rho-1$ if and only if the string starts with a $b$ and ends with an $a$.)

In this section we prove the main result of the paper, which is given in the following theorem:

Theorem 4. There is an algorithm which reconstructs a binary string $\mathbf{s}$ of length $n$ using $O(n)$ many $R C$-subsequence queries of length at most $\left\lceil\frac{2}{3}(n+1)\right\rceil$.

Notice that this is tight w.r.t. the lower bound of Fact 1 in all cases except where $n$ is a multiple of 3 . Even for these $n$, a gap of 1 unit is only necessary in the special case $A=\frac{2}{3} n$. In all other cases, our analysis resists the stricter bound of $T=\left\lceil\frac{2}{3} n\right\rceil$.

The proof of the theorem is by examining four cases separately. Recall that $A=|\mathbf{s}|_{a}, B=|\mathbf{s}|_{b}$, and $T=\frac{2}{3}(n+1)$. The four cases are: $1 . A \geq T, 2 . T>A>B$, 3. $A=B$ and $s_{1}=s_{n}$, and $4 . A=B$ and $s_{1} \neq s_{n}$. The following simple lemma will be used to distinguish these cases.

Lemma 5. Let $\mathbf{s}$ be a string of length at least 8 over $\{a, b\}, T=\left\lceil\frac{2}{3}(n+1)\right\rceil$, and $A=|\mathbf{s}|_{a} \geq|\mathbf{s}|_{b}$. Then,

1. using $O(\log n) R C$-subsequence queries of length at most $T$, it is possible to determine the exact value of $A=|\mathbf{s}|_{a}$ if $A<T$, or to establish the fact that $A \geq T$.

2. Moreover, if $A<T$, then it can be determined whether $\mathbf{s}$ starts and ends with the same character; furthermore, unless $A=\frac{n}{2}$ and $s_{1}=s_{n}$, we can determine $s_{1}$ and $s_{n}$. Altogether we require at most 3 additional $R C$-subsequence queries of length at most $T$.

Proof. 1. Binary search for $A$, using queries of the form $a^{\chi}$, for $\chi \in\left[\frac{n}{2}, T\right]$, will either return the exact value of $A$ (if $A<T$ ), or will exit with the maximum size query $a^{T} \prec_{\mathrm{RC}} \mathbf{s}$, thus showing that $A \geq T$.

2. Notice that if $A=B=\frac{n}{2}$, then the query $\mathbf{t}=a b^{\frac{n}{2}} a$ will return yes if and only if $s_{1}=s_{n}$. If $s_{1}=s_{n}$, then, due to the complete symmetry, we cannot determine the exact nature of $s_{1}$ and $s_{n}$. Otherwise, either $T>A=B$ and $s_{1} \neq s_{n}$, or $T>A>B$. In either case, the query $b a^{A}$ has length at most $T$ and will answer positively if and only if $s_{1}=b$. Likewise, the query $a^{A} b$ will answer positively if and only if $s_{n}=b$.

Example 6. Let $\mathbf{s}_{1}=a a b a b b b a$. Then $\tilde{\mathbf{s}}_{1}=b a a a b a b b$. The query $a b^{4} a$ will return yes and we can only determine that the first and last characters are equal, but not what they are. Instead, for $\mathbf{s}_{2}=a a b a b b a b$, we have $\tilde{\mathbf{s}}_{2}=a b a a b a b b$, the query $a b^{4} a$ will return no, and since the query $a b^{4}$ is positively answered, we know that the first character is $a$ (and thus the last character is $b$ ). 
Given a string $\mathbf{s}$ and a subsequence $\mathbf{t}$ of $\mathbf{s}$, we say that $\mathbf{t}$ fixes the direction of $\mathbf{s}$ if $\mathbf{t} \nprec \tilde{\mathbf{s}}$. If $\mathbf{t}$ fixes the direction of $\mathbf{s}$ then for any $\mathbf{t}^{\prime} \prec \mathbf{s}$, such that $\mathbf{t} \prec \mathbf{t}^{\prime}$ we also have that $\mathbf{t}^{\prime}$ fixes the direction of $\mathbf{s}$. In general, we shall try to identify $\mathbf{s}$ by first finding some sequence $\mathbf{t}$ which fixes the direction of $\mathbf{s}$ or $\tilde{\mathbf{s}}$ and then extending this $\mathbf{t}$. The importance of "direction-fixing" is that once we have found $\mathbf{t}$ which fixes the direction of $\mathbf{s}$, by asking queries about super-sequences of $\mathbf{t}$ we are sure that the answers to our queries are only about $\mathbf{s}$ and not its reverse complement.

The following two statements formalize two simple facts which will be used repeatedly in the following, thus, for the sake of completeness, we formally state and prove them here. Let $\mathbf{s}$ be fixed for the rest of this section.

Lemma 7. Let $\mathbf{t}=t_{1} \ldots t_{m}$ be a sequence which fixes the direction of $\mathbf{s}$. Fix a character $c \in \Sigma$. For each $i=1, \ldots, m+1$, let $\gamma_{i}=\min \left\{\max \left\{j \mid t_{1} \ldots t_{i-1} c^{j} t_{i} \ldots t_{m} \prec\right.\right.$ $\mathbf{s}\}, T-m\}$. Then, for each $i=1, \ldots, m+1$, we can determine $\gamma_{i}$ using $2 \log \gamma_{i}+1$ queries, or alternatively, using $\gamma_{i}+1$ queries. In particular, we can determine all $\gamma_{i}$ using at most $m+1+\sum_{i=1}^{m+1} \gamma_{i}$ queries.

Proof. We can determine all the values $\gamma_{i}$ either with one-sided binary search, using $2 \log \gamma_{i}+1$ queries, or with linear search, using $\gamma_{i}+1$ queries.

Example 8. Note that the lemma only assumes that $\mathbf{t}$ fixes the direction of $\mathbf{s}$, but not that the positions in $\mathbf{s}$ to which the characters of $\mathbf{t}$ are matched are also fixed. Consider the following example. Let $\mathbf{s}=a^{10} b a^{10} b a^{10}$. Then $\mathbf{t}=a a a$ fixes the direction of $\mathbf{s}$. For $c=b$, we get $\gamma_{1}=\gamma_{4}=0$ and $\gamma_{2}=\gamma_{3}=1$. For $c=a$, we have $\gamma_{i}=\min (27,22)=22$ for all $i($ since $T=22)$.

The next lemma says that if there are large $a$-runs or large $b$-runs, then there cannot be many runs.

Lemma 9. Let $\mathbf{s}=a^{x_{1}} b^{y_{1}} \ldots a^{x_{t}} b^{y_{t}}$. Assume that there are $1 \leq i_{1}<i_{2}<\cdots<$ $i_{q} \leq t$ and $k \geq 0$, such that $x_{i_{j}} \geq T-B-k$ (resp. $y_{i_{j}} \geq T-A-k$ ) for each $j=1, \ldots, q$, and for at least one value of $j$ it holds that $x_{i_{j}}>T-B-k$ (resp. $\left.y_{i_{j}}>T-A-k\right)$. Then

$\rho_{a} \leq n-B-q(T-B-k-1)-1 \quad\left(\right.$ resp. $\left.\rho_{b} \leq n-A-q(T-A-k-1)-1\right)$.

Proof. We limit ourselves to showing the argument for $\rho_{a}$. Since each run counted by $\rho_{a}$ has at least one $a$, we have the desired inequality:

$$
n-B=A \geq \sum_{j=1}^{q} x_{i_{j}}+\rho_{a}-q \geq q(T-B-k)+1+\rho_{a}-q .
$$

\subsection{The case where $A \geq T$}

Since $A \geq T=\left\lceil\frac{2}{3}(n+1)\right\rceil$, we have that $B \leq \frac{n}{3}-\frac{2}{3}$, so $2 B+1=2(n-A)+1 \leq$ $\frac{2}{3}(n+1) \leq T$. This implies that we can ask queries which include $B+1$ many $a$ 's and up to $B$ many $b$ 's. Let $\beta=\left\lceil\frac{n}{3}-\frac{2}{3}\right\rceil$, and $\mathbf{t}=a^{\beta+1}$. We have $B \leq \beta$ and, therefore, $\mathbf{t}$ fixes the direction of $\mathbf{s}$. Notice also that $B+\beta+1 \leq T$. 
By Lemma 7, with $\mathbf{t}=a^{\beta+1}$, we can find $L=\max \left\{j \mid b^{j} a^{\beta+1} \prec \mathbf{s}\right\}$ with $O(\log L)$ queries. Likewise, with $\mathbf{t}=b^{L} a^{\beta+1}$ we can find $R=\max \left\{j \mid b^{L} a^{\beta+1} b^{j} \prec\right.$ s\}, with $O(\log R)$ queries.

Notice that in $\mathbf{s}$, between the left-most $L$ many $b$ 's and the right-most $R$ many $b$ 's, there may be more than $\beta+1$ many $a$ 's. More precisely, with reference to (1), the previous queries guarantee that there are $1 \leq i \leq j \leq \rho$ such that $\sum_{k=1}^{i-1} y_{k}=L$ and $\sum_{k=j}^{\rho} y_{k}=R$. Let $\mathbf{w}$ be the substring of $\mathbf{s}$ between the $L$ left-most and the $R$ right-most $b$ 's, i.e., $\mathbf{w}=a^{x_{i}} b^{y_{i}} \cdots a^{x_{j}}$. Moreover, let $\mathbf{s}_{\text {left }}$ and $\mathbf{s}_{\text {right }}$ be such that $\mathbf{s}=\mathbf{s}_{\text {left }} \mathbf{w s}_{\text {right }}$. We know that $\left|\mathbf{s}_{\text {left }}\right|_{b}=L,\left|\mathbf{s}_{\text {right }}\right|_{b}=R$, and $|\mathbf{w}|_{a} \geq \beta+1$. We will first determine all but the first $a$-run of $\mathbf{w}$ and all of its $b$-runs, in particular yielding the exact value of $B$. Then we determine $\mathbf{s}_{\text {left }}$ and $\mathbf{s}_{\text {right }}$. For any $a$-runs that have length at least $T-B$, their exact value will be determined during the final stage.

We have $a^{\beta+1} \prec \mathbf{w}$, and by the definition of $L$ and $R$ we also have that $\sum_{k=i+1}^{\rho} x_{k} \leq \beta$ and $x_{i}>\sum_{k=j+1}^{\rho} x_{k}$. It follows that, for $\chi=1,2,3 \ldots, \beta$, the query $b^{L} a^{\chi} b a^{\beta+1-\chi} b^{R}$ answers negatively as long as $\sum_{k=i+1}^{j} x_{k}<\beta+1-\chi$. Let $\chi^{*}$ be the first value for which the answer to this query is yes, and $\chi^{*}=\beta+1$ if the answer is no for all values of $\chi$. It is easy to see that $\chi^{*}=\beta+1-\sum_{k=i+1}^{j} x_{k}$. In particular, $\chi^{*}=\beta+1$ if and only if if $\mathbf{w}$ does not contain any $b$ 's. In this case, set $\mathbf{w}^{\prime}=a^{\beta+1}$.

If $\chi^{*} \leq \beta$, define $\mathbf{t}=b^{L} a^{\chi^{*}} b a^{\beta+1-\chi^{*}} b^{R}$. By Lemma 7 , with $\mathbf{t}$ we can find the value of $y_{k}$ for each $k=i, \ldots, j-1$. As a side effect, we also determine the value of $x_{k}$ for $k=i+1, \ldots, j$. Now we know that $b^{L} \mathbf{w}^{\prime} b^{R} \prec \mathbf{s}$, where $\mathbf{w}^{\prime}=a^{\chi^{*}} b^{y_{i}} a^{x_{i+1}} \ldots b^{y_{j-1}} a^{x_{j}}$. In other words, we know $\mathbf{w}$ except for its first $a$-run, which may be longer than $\chi^{*}$. We also know $B$, the number of $b$ 's of $\mathbf{s}$.

Now we turn to $\mathbf{s}_{\text {right }}$. Let us denote by $\mathbf{w}^{\prime}-a^{\ell}$ an arbitrary sequence obtained by removing exactly $\ell$ many $a$ 's from $\mathbf{w}^{\prime}$ and leaving the rest as it is. Now we can use queries of the form $b^{L}\left(\mathbf{w}^{\prime}-a^{\ell}\right) b^{r} a^{\ell} b^{R-r}$ with $r=1, \ldots, R$ and $\ell=1,2,3 \ldots$, in order to determine the values of $x_{k}$, for each $k=j+1, \ldots, \rho$. To see this, it is enough to notice that each such query contains $\beta+1$ many $a$ 's, therefore it can only be a subsequence of $\mathbf{s}$ and not of $\tilde{\mathbf{s}}$. Moreover, we notice that in order to determine $x_{k}$ we need to receive a positive answer to the query $b^{L}\left(\mathbf{w}^{\prime}-a^{x_{k}}\right) b^{\sum_{\ell=j}^{k-1} y_{\ell}} a^{x_{k}} b^{R-\sum_{\ell=j}^{k-1} y_{\ell}}$ and a negative answer to the query $b^{L}\left(\mathbf{w}^{\prime}-a^{x_{k}-1}\right) b^{\sum_{\ell=j}^{k-1} y_{\ell}} a^{x_{k}+1} b^{R-\sum_{\ell=j}^{k-1} y_{\ell}}$. Because of $\sum_{k=j+1}^{\rho} x_{k}<\beta+1$, both these queries have length not larger than $T$. Again, by determining $x_{k}$ for each $k=j+1, \ldots, \rho$, we also determine $y_{k}$ for each $k=j+1, \ldots, \rho$.

By an analogous procedure, we can determine $\mathbf{s}_{\text {left }}$ and the first $a$-run of $\mathbf{w}$, i.e. all the values $x_{k}$, for $k=1, \ldots, i$, where $x_{k} \leq T-B$. Again, in this process, we also determine the size of the runs of $b$ 's, i.e., the $y_{k}$, for each $k=1, \ldots, i-1$.

Finally, we compute the size of the $a$-runs in $\mathbf{s}$ that are larger than $T-B$. Notice that for at most two indices we can have $x_{k} \geq T-B$, for otherwise their total sum would be larger than $n$, the total length of the string. If there is exactly one such $x_{k}$, then we can compute it as $x_{k}=n-B-\sum_{\ell \neq k} x_{\ell}$. Otherwise, let $1 \leq i_{1}<i_{2} \leq i$ be such that $x_{i_{1}}, x_{i_{2}} \geq T-B$. Then it must hold 
that $n-B-\sum_{\ell \neq i_{1}, i_{2}} x_{\ell}=2(T-B)$, and thus, $x_{i_{1}}, x_{i_{2}}=T-B$. Otherwise, we would have that $x_{1}+x_{2}>2(T-B)$, and using Lemma 9 , with $k=0$, we can then conclude that $\rho_{a} \leq n-2 T+B+1 \leq-1$, a contradiction.

Notice that we use at most one query per character of s plus at most one query for each run of $\mathbf{s}$. Therefore, it total we have $O\left(\sum_{i}\left(x_{i}+1\right)+\sum_{i}\left(y_{i}+1\right)\right)=O(n)$.

\subsection{The case $T>A>B$}

By Lemma 7 with $\mathbf{t}=a^{A}$ and $c=b$, with $O(n)$ queries we can determine exactly $y_{k}$ for each $k$ such that $y_{k}<T-A$. In the process, we also find out exactly $x_{k}$ for each $k=1, \ldots, \rho_{a}$. The only problem now is to determine those runs of $b$ 's which have length at least $T-A$.

Let $i_{1}, \ldots, i_{q}$ be the $q$ distinct indices of the runs of $b$ 's such that $y_{i_{j}} \geq T-A$, so we have not yet been able to determine their exact value. Clearly, if $q=1$, we can compute $y_{i_{1}}=B-\sum_{\ell \neq i_{1}} y_{\ell}$. Likewise, if $B-\sum_{\ell \neq i_{1}, \ldots, i_{q}} y_{\ell}=q(T-A)$, then we know $y_{i_{j}}=T-A$ for all $i_{j}$. Otherwise, it must hold that $\sum_{j=1}^{q} y_{i_{j}}>q(T-A)$. Let $y_{i_{1}} \geq y_{i_{2}} \geq \cdots \geq y_{i_{q}}$ and $\alpha>0$ such that $y_{i_{1}}=T-A+\alpha$. We have

$$
\rho_{b} \leq B-\left(y_{i_{1}}+y_{i_{2}}\right)+2=n-A-(T-A+\alpha)-y_{i_{2}}+2 \leq \frac{n}{3}+\frac{4}{3}-\alpha-y_{i_{2}} .
$$

Now, consider the sequence $\mathbf{t}_{\chi}=(a b)^{i_{2}-1} a b^{\chi}(a b)^{\rho_{b}-i_{2}}$. For each $\chi=T-A+$ $1, T-A+2, \ldots, y_{i_{2}}+1$, such a string has length at most $T$, since we have

$\left|\mathbf{t}_{\chi}\right|=2 \rho_{b}+\chi-1 \leq 2 \rho_{b}+y_{i_{2}} \leq \frac{2 n}{3}+\frac{8}{3}-2 \alpha-2 y_{i_{2}}+y_{i_{2}}=\frac{2 n}{3}+\frac{8}{3}-2 \alpha-y_{i_{2}} \leq T-1$,

where the last inequality follows from the fact that $\alpha, y_{i_{2}} \geq 1$.

We will finish the proof for the case $T>A>B$ by distinguishing four cases according to whether $s_{1}=s_{n}$ and whether $s_{1}=a$ or $s_{1}=b$. (Note that due to the assumption $A>B$ we cannot assume w.l.o.g. the identity of the first character.)

Case 1. If $s_{1}=s_{n}=b$, then $\rho_{b}=\rho$, we can remove the first $a$ from $\mathbf{t}_{\chi}$, and the new query fixes the direction of $\mathbf{s}$. This query has length at most $T$, so we can identify $y_{i_{2}}$. By the same argument, we can also identify $y_{i_{j}}$, for each $j=3, \ldots, q$, since $y_{i_{j}} \leq y_{i_{2}}$, for each such $j$. Finally we can determine $y_{i_{1}}$ by subtraction.

Case 2. If $s_{1}=s_{n}=a$, then $\rho_{b}=\rho-1$. Now we have to add an $a$ at the end to get a query which fixes the direction of $\mathbf{s}$, and its length is again at most $T$. The argument is then analogous to Case 1.

Case 3. Let $s_{1} \neq s_{n}$ and $s_{1}=b$. This case is analogous to Case 4 . below, replacing $\mathbf{t}_{\chi}$ by $\mathbf{u}_{\chi}=(b a)^{i_{2}-1} b^{\chi} a(b a)^{\rho_{b}-i_{2}}$ and all following sequences accordingly.

Case 4. As the final case we have $s_{1} \neq s_{n}$ and $s_{1}=a$, so $\rho_{b}=\rho$. We will now look at the value of $X:=x_{\rho-i_{2}+1}$. Note that any query $\mathbf{t}_{\chi}$ with $\chi \leq X$ would 
answer yes because it would be interpreted as $\tilde{\mathbf{t}}_{\chi}$. Notice that we know the value of $X$. If $X<T-2 \rho$, then we ask query $\mathbf{t}_{\chi}$ for $\chi=X+1$. If the answer is yes, we continue with $X+2, X+3 \ldots$ until we receive the first no, and we are done, since the last $\chi$ where $\mathbf{t}_{\chi}$ answered positively was equal to $y_{i_{2}}$. By (2), these queries do not exceed the threshold.

Otherwise, if the query $\mathbf{t}_{X+1}$ answered $n o$ or if $X>T-2 \rho$, then we know that $y_{i_{2}} \leq X$. In this case, we use the following queries to determine $y_{i_{2}}$.

Let w.l.o.g. $i_{2} \leq \rho-i_{2}+1$ (otherwise exchange the roles of $i_{2}$ and $\rho-i_{2}+1$ in the formulas below). Define $\mathbf{t}_{\xi}^{\prime}=(a b)^{i_{2}-1} a^{\xi}(b a)^{\rho-2 i_{2}+1} b^{\xi+1}(a b)^{i_{2}-1}$. One can verify that for each $\xi=T-A, T-A+1, \ldots, y_{i_{2}}$, we have

$$
\left|\mathbf{t}_{\xi}^{\prime}\right| \leq 2 \rho_{b}+2 y_{i_{2}}-1 \leq \frac{2 n}{3}+\frac{2}{3}-2 \alpha+1 \leq T+1-2 \alpha \leq T-1,
$$

where the last inequality follows from the fact that $\alpha \geq 1$.

We can ask queries $\mathbf{t}_{\xi}^{\prime}$ until either we receive a negative answer or we cannot enlarge it further because it would violate the bound $T$. The largest value of $\xi$ for which we receive a positive answer to query $\mathbf{t}_{\xi}^{\prime}$ correctly gives the value of $y_{i_{2}}$. Clearly this is true if we also receive a negative answer, for the next larger value. If, instead, we had to stop because of the bound $T$, we can be sure that $\xi=y_{i_{2}}$, because if $y_{i_{2}}>\xi$, then this would contradict the inequality (3).

We ask at most one query per character plus one query per run, except for Case 4 , where we might use two queries per character of the $y_{i_{2}}$ 'th run of $b$ 's. Altogether, we have that the total number of queries is $O(n)$.

\subsection{The case $T>A=B=\frac{n}{2}, s_{1}=s_{n}$}

We assume w.l.o.g. that the string starts and ends in $a$. Therefore, with reference to (1), in this section we have $y_{\rho}=0$ and our string looks like this:

$$
\mathbf{s}=a^{x_{1}} b^{y_{1}} a^{x_{2}} b^{y_{2}} \ldots a^{x_{\rho-1}} b^{y_{\rho-1}} a^{x_{\rho}},
$$

with all $x_{i}, y_{i}>0$, i.e., it includes $\rho=\rho_{a}$ runs of $a$ 's and $\rho-1=\rho_{b}$ runs of $b$ 's.

By Lemma 7 with $\mathbf{t}=a b^{\frac{n}{2}}$ we can exactly determine $x_{k}$ (run of $a$ 's) for each $k$, such that $x_{k}<T-\frac{n}{2}-1 \leq \frac{n}{6}-\frac{1}{3}$. In this process, we determine exactly $y_{k}$, for each $k=1, \ldots, \rho_{b}$.

Let $1 \leq i_{1}<i_{2}<\cdots<i_{q} \leq \rho_{a}$ be all the indices of the runs of $a$ 's whose length we have not been able to determine exactly, i.e., such that $x_{i_{j}} \geq T-\frac{n}{2}-1$. By $A=\frac{n}{2}$, we have that $q \leq 3$. In fact, the only interesting cases are $q=2$ and $q=3$, since, for $q=1$ we can determine the only missing $x_{i_{1}}$, as the difference between $A$ and the sum of the remaining $x_{k}$ 's.

For $q=3$, by Lemma 9, we have $\rho_{a} \leq 3$, thus it follows that $\rho_{a}=3$. Let $\mathbf{t}=a b a b a$, and $c=a$. By Lemma 7 we can determine each $x_{k}$, such that $x_{k} \leq T-5$. Suppose that for all $k=1,2,3$, it holds that $x_{k} \geq T-5$. Since there must exist one run of $a$ 's of length $\leq \frac{n}{6}$, we have that $n \leq 9$, whence $A \leq 4$, implying that the only possible case is to have two runs of $a$ 's of length 1 and 
one run of $a$ 's of length 2. Direct inspection shows that in this case we can easily reconstruct the whole string with $T$-bounded queries.

Finally, if $q=2$, by Lemma 9, we have $\rho_{a} \leq \frac{n}{6}+\frac{5}{3}$. We can now use query $\mathbf{t}_{1}=(a b)^{i_{1}-1} a^{T-\frac{n}{2}-1+\chi}(b a)^{\rho-i_{1}}$, for $\chi=1,2,3, \ldots$, until we receive a negative answer, then $x_{i_{1}}=T-\frac{n}{2}-1+\chi-1$. If we never receive a negative answer and the query becomes of length $T$, we can resort to the query $\mathbf{t}_{2}=(a b)^{i_{2}-1} a^{T-\frac{n}{2}-1+\chi}(b a)^{\rho-i_{2}}$, for $\chi=1,2, \ldots$, and proceed analogously. It is easy to see that we cannot have that both $\mathbf{t}_{1}$ and $\mathbf{t}_{2}$ exceed the threshold $T$; the other value can then be determined by difference.

We have used $O(\log n+A)=O(n)$ many queries.

\subsection{The case $T>A=B=\frac{n}{2}, s_{1} \neq s_{n}$}

Recall that by Lemma 5 , in this case we can exactly determine $s_{1}$ and $s_{n}$. Let us assume w.l.o.g. that $s_{1}=a$ and $s_{n}=b$. (Otherwise, rename the characters.) Then the string $\mathbf{s}$ has the following shape

$$
\mathbf{s}=a^{x_{1}} b^{y_{1}} a^{x_{2}} b^{y_{2}} \ldots a^{x_{\rho-1}} b^{y_{\rho-1}} a^{x_{\rho}} b^{y_{\rho}} .
$$

In particular, it starts with a run of $a$ 's and ends with a run of $b$ 's.

We need some more notation. For each $i=1,2, \ldots, 2 \rho$, we use $r_{i}$ to denote the size of the $i$ 'th run in $\mathbf{s}$ starting from the left. I.e., we have $x_{i}=r_{2 i-1}$ and $y_{i}=r_{2 i}$ for each $i=1, \ldots, \rho$. Also we denote by $m_{i}=\min \left\{r_{i}, r_{2 \rho-i+1}\right\}$ and by $M_{i}=\max \left\{r_{i}, r_{2 \rho-i+1}\right\}$. We use the following technical lemma, whose proof can be found in the Appendix.

Lemma 10. Fix $i<\rho$ and assume that for each $k=1, \ldots, i-1$, we know $r_{k}$ and $r_{2 \rho-k+1}$ and it holds that $r_{k}=r_{2 \rho-k+1}<T-\frac{n}{2}$. Then we can determine $m_{i}$ and $\min \left\{M_{i}, T-\frac{n}{2}\right\}$, asking at most $\max \left\{m_{i}, \min \left\{M_{i}, T-\frac{n}{2}\right\}\right\}$ queries.

Now, let us consider the largest $k \geq 1$ such that $r_{j}=r_{2 \rho-j+1}<T-\frac{n}{2}$ for each $j<k$. Note that by repeated application of Lemma 10, we can determine all these $r_{j}$ 's. Assume w.l.o.g. that $k$ is odd and let $i=\lceil k / 2\rceil$. Then we can write:

$$
\mathbf{s}=\mathbf{u} a^{x_{i}} \mathbf{s}^{\prime} b^{y_{\rho-i+1}} \tilde{\mathbf{u}}
$$

where $\mathbf{u}=a^{x_{1}} b^{y_{1}} \ldots a^{x_{i-1}} b^{y_{i-1}}$ is known, and the string $\mathbf{s}^{\prime}$ is still unknown. Note that also the two values $\min \left\{x_{i}, y_{\rho-i+1}\right\}$ and $\min \left\{\max \left\{x_{i}, y_{\rho-i+1}\right\}, T-\frac{n}{2}\right\}$ are known (again by application of Lemma 10). Moreover, for determining these two values and string $\mathbf{u}$, we have used a number of queries linear in $2|\mathbf{u}|+$ $\min \left\{\max \left\{x_{i}, y_{\rho-i+1}\right\}, T-\frac{n}{2}\right\}$.

According to the magnitude of $x_{i}$ and $y_{\rho-i+1}$, we will enter one of the following three cases, where we will assume, w.l.o.g., that $x_{i} \leq y_{\rho-i+1}$. (The case where $y_{\rho-i+1}<x_{i}$ is symmetric.) We illustrate the situation in Figure 1. 


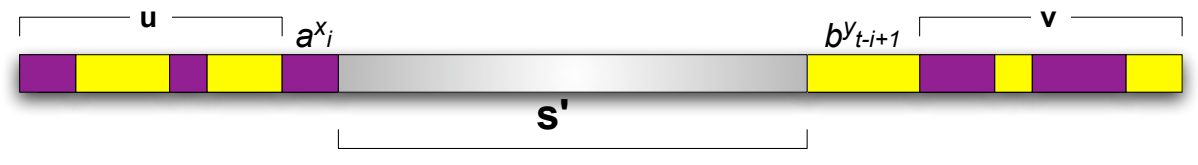

Fig. 1. The case where $|\mathbf{s}|_{a}=|\mathbf{s}|_{b}$ and $s_{1} \neq s_{n}$. We determine $\mathbf{s}$ by first finding the first assymetry in $\mathbf{s}\left(x_{i} \neq y_{\rho-i+1}\right)$, and then extending queries for $\mathbf{s}^{\prime}$, which has fewer $b$ 's than $a$ 's. Note that up to index $i$, string $\mathbf{s}$ is perfectly symmetric, i.e. we have $\mathbf{v}=\tilde{\mathbf{u}}$.

The case $A=B=\frac{n}{2}, s_{1} \neq s_{n}, x_{i}, y_{\rho-i+1}<T-\frac{n}{2}$. With reference to (4), we can use a recursive argument to show how to determine $\mathbf{s}^{\prime}$. Let $n^{\prime}=\left|\mathbf{s}^{\prime}\right|$. Note that $\left|\mathbf{s}^{\prime}\right|_{a}>\left|\mathbf{s}^{\prime}\right|_{b}$ and that $\mathbf{s}^{\prime}$ starts with a $b$ and ends with an $a$.

Let $\mathbf{t}^{\prime}$ be a query for $\mathbf{s}^{\prime}$ : Since $\left|\mathbf{s}^{\prime}\right|_{a}>\left|\mathbf{s}^{\prime}\right|_{b}$, such queries were defined by one of the previous cases (Section 4.1 or 4.2 ). Let $\mathbf{t}_{+}^{\prime}$ be the query obtained by adding to $\mathbf{t}^{\prime}$ an initial $b$, if $\mathbf{t}^{\prime}$ does not begin with $b$, and a final $a$, if $\mathbf{t}^{\prime}$ does not end with an $a$. Define a query $\mathbf{t}$ for $\mathbf{s}$ in the following way:

$$
\mathbf{t}=a^{|\mathbf{u}|_{a}} \mathbf{t}_{+}^{\prime} a^{|\mathbf{u}|_{b}}
$$

Lemma 11. Let $\mathbf{t}$ be defined as in Eq. (5). Then, it holds that

$$
\begin{aligned}
& \text { 1. } \mathbf{t} \prec_{\mathrm{RC}} \mathbf{S} \text { if and only if } \mathbf{t}^{\prime} \prec_{\mathrm{RC}} \mathbf{s}^{\prime} \text {. } \\
& \text { 2. If }\left|\mathbf{t}^{\prime}\right| \leq \frac{2\left(n^{\prime}+1\right)}{3} \text {, then }|\mathbf{t}| \leq \frac{2(n+1)}{3} \text {. }
\end{aligned}
$$

Thus it follows that we can use the analysis of the previous sections to prepare a sequence of queries on $\mathbf{s}$ which is (i) linear in $\left|\mathbf{s}^{\prime}\right|$ and (ii) allows us to determine the substring $\mathbf{s}^{\prime}$ of $\mathbf{s}$. Once this is accomplished, the whole $\mathbf{s}$ can be fully determined (up to reverse complement).

The case $A=B=\frac{n}{2}, s_{1} \neq s_{n}, x_{i}, y_{\rho-i+1} \geq T-\frac{n}{2}$. Notice that, because of the assumption $n \geq 8$ and $T-\frac{n}{2} \leq x_{i}, y_{\rho-i+1}$, it follows that $x_{i}+y_{\rho-i+1} \geq 4$. We have $\left|\mathbf{s}^{\prime}\right|=n-2|\mathbf{u}|-x_{i}-y_{\rho-i+1}$. This implies

$$
\left|\mathbf{s}^{\prime}\right|+|\mathbf{u}|+2 \leq n-x_{i}-y_{\rho-i+1}+2+|\mathbf{u}| \leq 2 n-2 T+2-|\mathbf{u}| \leq \frac{2 n}{3}+\frac{2}{3}-|\mathbf{u}| \leq T .
$$

Thus we can adapt the strategy we described in Section 3 for unbounded RC-reconstruction to determine $\mathbf{s}^{\prime}$ and then, by subtraction, also $x_{i}$ and $y_{\rho-i+1}$. We proceed as follows: Suppose that in the strategy for reconstructing $\mathbf{s}^{\prime}$, in the unbounded-query case, we ask a question $\mathbf{t}^{\prime}$, starting with $b$ and ending with $a$. Then we will ask query $\mathbf{t}=a^{|\mathbf{u}|_{a}+1} \mathbf{t}^{\prime} b a^{|\mathbf{u}|_{b}}$. It is not hard to see that such $\mathbf{t}$ answers positively on $\mathbf{s}$ if and only if $\mathbf{t}^{\prime}$ answers positively on $\mathbf{s}^{\prime}$. By (6), $\mathbf{t}|=| \mathbf{t}^{\prime}|+2+| \mathbf{u} \mid \leq T$.

The only requirement is that $\mathbf{t}^{\prime}$ begin with $b$ and end with $a$. However, the strategy in Section 3 can be easily adapted to this case, under the assumption 
that the string to be reconstructed begins with $b$ and ends with $a$, a condition that holds for $\mathbf{s}^{\prime}$. (Notice, in fact, that because the query size is unbounded, any query in the strategy in Section 3 can be safely extended by an arbitrary prefix and/or suffix of the string we are trying to reconstruct.)

Finally, once we have reconstructed $\mathbf{s}^{\prime}$ we can determine $\max \left\{x_{i}, y_{\rho-i+1}\right\}$ as $\frac{n}{2}-\left|\mathbf{s}^{\prime}\right|_{b}-|\mathbf{u}|$. (Recall that we have assumed w.l.o.g. that $x_{i} \leq y_{\rho-i+1}$; in fact, now that we know $\mathbf{s}^{\prime}$, we can determine whether this is the case: we have $x_{i} \leq y_{\rho-i+1}$ if and only if $\left|\mathbf{s}^{\prime}\right|_{a} \geq\left|\mathbf{s}^{\prime}\right|_{b}$.)

The case $A=B=\frac{n}{2}, s_{1} \neq s_{n}, x_{i}<T-\frac{n}{2}, y_{\rho-i+1} \geq T-\frac{n}{2}$. In order to determine $\rho$ and $x_{i+1}, \ldots, x_{\rho-i+1}$, we can use the query

$$
\mathbf{t}_{\chi}=a^{|\mathbf{u}|_{a}+x_{i}} b a^{\chi} b a^{\frac{n}{2}-|\mathbf{u}|_{a}-x_{i}-\chi}
$$

as follows. Under the standing hypothesis, we have $x_{i}<\frac{2(n+1)}{3}-\frac{n}{2} \leq y_{\rho-i+1}$. The above query $\mathbf{t}_{\chi}$ has size $\frac{n}{2}+2 \leq T$, for any $n \geq 8$. Moreover, the fact that $x_{i}<y_{\rho-i+1}$ guarantees that if $\mathbf{t}_{\chi} \prec \mathbf{s}$ then $\mathbf{t}$ fixes the direction of $\mathbf{s}$. To see this, with reference to (4), it is enough to observe that in this case, in $\mathbf{s}$ there are more $a$ 's following the first $b$ of $\mathbf{s}^{\prime}$ than there are $b$ 's preceeding the last $a$ of $\mathbf{s}^{\prime}$.

We use the query $\mathbf{t}_{\chi}$ as follows: We ask $\mathbf{t}_{\chi}$ for each $\chi=1,2,3 \ldots$, until we get the first positive answer. Let $\chi_{1}$ be the minimum value of $\chi$ for which the answer is positive. It is not hard to see that this implies $x_{i+1}=\chi_{1}$. We now continue asking query $\mathbf{t}_{\chi}$ for each $\chi=\chi_{1}+1, \chi_{1}+2, \ldots$. Let $\chi_{2}$ be the minimum value of $\chi$ for which we get a new positive answer. Again, this implies that $x_{i+2}=\chi_{2}-\chi_{1}$. More generally, for each $j=1, \ldots, \rho-i+1$, let $\chi_{j}$ be the value of $\chi$ when we receive the $i$ th positive answer. Then, we have $x_{i+j}=\chi_{j}-\chi_{j-1}$ (where we set $\chi_{0}=0$ for sake of definiteness).

Note, however, that at this point we do not know $\rho$. We continue asking $\mathbf{t}_{\chi}$ as long as $\frac{n}{2}-|\mathbf{u}|_{a}-x_{i}-\chi>|\mathbf{u}|_{b}$, or equivalently, $\chi<\frac{n}{2}-|\mathbf{u}|-x_{i}$. This way we determine $x_{j}$, for $j=i+1, \ldots, \rho-i+1$ and, in particular, we determine $\rho$.

Now by Lemma 7 , with $\mathbf{t}=a^{|\mathbf{u}|_{a}+x_{i}} b a^{\frac{n}{2}-x_{i}-|\mathbf{u}|_{a}}$, we can determine exactly $y_{j}$, for each $j=i, \ldots, \rho-i$ such that $y_{j}<T-\frac{n}{2}$, or, otherwise, establish the fact that $y_{j} \geq T-\frac{n}{2}$. As in the previous cases, it now remains to determine the exact values of those runs with length at least $T-\frac{n}{2}$.

Let $i_{1}, \ldots, i_{q}$, be such that $y_{i_{j}} \geq T-\frac{n}{2}$, for each $j=1, \ldots, q$. We can also assume that for at least one $1 \leq j \leq q$ it holds that $y_{i_{j}}>T-\frac{n}{2}$, for otherwise we can identify this situation by the fact that $n-\sum_{\ell \notin\left\{i_{1}, \ldots, i_{q}\right\}} y_{\ell}=q\left(T-\frac{n}{2}\right)$, whence we have $y_{i_{j}}=T-\frac{n}{2}$, for each $j$.

For each $j$ such that $y_{i_{j}} \geq T-\frac{n}{2}$ and whose value is not determined yet, we use a query of the form:

$$
\mathbf{t}_{\chi}=(a b)^{i_{j}-1} a b^{\chi}(a b)^{\rho-i-i_{j}} a b^{x_{i}+1}(a b)^{i-1},
$$

increasing $\chi$ until we get the first positive answer. It remains to show that each of these queries has length smaller or equal to $T$. 
We have that $\left|\mathbf{t}_{\chi}\right|=2 \rho+x_{i}+\chi-1$. To see that this is smaller or equal to $T$ for each $\chi \leq y_{i_{j}}$, notice that $y_{i_{j}} \geq \frac{2(n+1)}{3}-\frac{n}{2}=\frac{n}{6}+\frac{2}{3}$. Further, by assumption, we have $y_{\rho-i+1}, y_{i_{j}} \geq \frac{n}{6}+\frac{2}{3}$, implying $t \leq \frac{n}{2}-\frac{2 n}{6}+\frac{2}{3}=\frac{n}{6}+\frac{2}{3} \leq y_{\rho-i+1}$. Thus, we have $\rho \leq y_{\rho-i+1}$. Moreover, recall that $\frac{n}{2}=B \geq y_{\rho-i+1}+\rho+y_{i_{j}}-2$. Putting it all together, we get

$\mathbf{t}_{y_{i_{j}}}=2 \rho+x_{i}+y_{i_{j}}-1 \leq \underbrace{y_{\rho-i+1}+\rho+y_{i_{j}}-1}_{\leq B+1=\frac{n}{2}+1}+x_{i} \leq \frac{n}{2}+\underbrace{x_{i}+1}_{\leq \frac{n}{6}+\frac{2}{3}}<\frac{2(n+1)}{3} \leq T$.

As can be readily seen, in all three subcases we use $O\left(\left|\mathbf{s}^{\prime}\right|\right)$ queries to determine $\mathbf{s}^{\prime}$, hence, altogether $O(|\mathbf{s}|)$ queries to complete the reconstruction.

\section{References}

1. A. Carpi and A. de Luca. Words and special factors. Theor. Comput. Sci., 259(12):145-182, 2001.

2. A. de Luca. On the combinatorics of finite words. Theor. Comput. Sci., 218(1):1339, 1999.

3. M. Dudík and L. J. Schulman. Reconstruction from subsequences. J. Comb. Theory, Ser. A, 103(2):337-348, 2003.

4. P. L. Erdős, P. Ligeti, P. Sziklai, and D. C. Torney. Subwords in reverse-complement order. In Combinatorial and Algorithmic Foundations of Pattern and Association Discovery, 2006.

5. G. Fici, F. Mignosi, A. Restivo, and M. Sciortino. Word assembly through minimal forbidden words. Theor. Comput. Sci., 359(1-3):214-230, 2006.

6. I. Krasikov and Y. Roditty. On a reconstruction problem for sequences. Journal of Combinatorial Theory, 77:344-348, 1997.

7. V. I. Levenshtein. Efficient reconstruction of sequences. IEEE Transactions on Information Theory, 47(1):2-22, 2001.

8. P. Pevzner. l-tuple DNA sequencing: Computer analysis. Journal of Biomolecular Structure and Dynamics.

9. C. Piña and C. Uzcátegui. Reconstruction of a word from a multiset of its factors. Theor. Comput. Sci., 400(1-3):70-83, 2008.

10. F. P. Preparata. Sequencing-by-hybridization revisited: The analog-spectrum proposal. IEEE/ACM Trans. Comput. Biology Bioinform., 1(1):46-52, 2004.

11. M.-P. Schützenberger and I. Simon. Combinatorics on Words, by M. Lothaire, chapter 6: Subwords. 1983.

12. I. Simon. Piecewise testable events. In Automata Theory and Formal Languages, pages 214-222. Springer, 1975.

13. S. Skiena and G. Sundaram. Reconstructing strings from substrings. Journal of Computational Biology, 2(2):333-353, 1995.

14. D. Tsur. Tight bounds for string reconstruction using substring queries. In APPROX-RANDOM, pages 448-459, 2005. 


\section{Appendix}

Fact 1 (Erdös et al., 2006 [4]). For any $n \geq 4$ there exist two distinct strings of size $n$ with exactly the same set of subsequences of length up to $\left\lceil\frac{2 n}{3}\right\rceil-1$.

Proof. Let us write $n$ as $n=3 k+r$, with $r \in\{0,1,2\}$. Let $\mathbf{s}_{1}=a^{2 k+r} b^{k}$ and $\mathbf{s}_{2}=a^{2 k+r-1} b^{k+1}$. It is not hard to see that we have $a^{2 k+r} \prec_{\mathrm{RC}} \mathbf{s}_{1}$ but $a^{2 k+r} \nprec_{\mathrm{RC}} \mathbf{s}_{2}$ whilst for any string $\mathbf{t} \prec_{\mathrm{RC}} \mathbf{s}_{1}$ such that $|\mathbf{t}| \leq 2 k+r-1$ it holds that $\mathbf{t} \prec_{\mathrm{RC}} \mathbf{s}_{2}$.

\section{Unbounded query size (Section 3)}

To prove Theorem 3, we first need an easy lemma, which extends Lemma 14 in [13] to our problem.

Lemma 12. Let $\mathbf{s}$ be an unknown string over the paired alphabet $\Sigma=\left\{a_{1}, \overline{a_{1}}, \ldots, a_{\delta}, \overline{a_{\delta}}\right\}$, (where we have explicitly specified the complementing pairs). Let $\mathbf{u}, \mathbf{v} \prec_{\mathrm{RC}} \mathbf{s}$ be two known RC-character-disjoint strings over $\Sigma$, i.e., no character of $\mathbf{u}$ or its pair occurs in $\mathbf{v}$, and vice versa. Then it is possible to construct a string $\mathbf{w}$ such that $\mathbf{u}, \mathbf{v} \prec_{\mathrm{RC}} \mathbf{w}$ and $\mathbf{w} \prec_{\mathrm{RC}} \mathbf{s}$ using at most $2(|\mathbf{u}|+|\mathbf{v}|+1)$ queries.

Proof. By definition, at least one of the four cases must hold: $\mathbf{u}, \mathbf{v} \prec \mathbf{s}, \tilde{\mathbf{u}}, \tilde{\mathbf{v}} \prec \mathbf{s}$, $\mathbf{u}, \tilde{\mathbf{v}} \prec \mathbf{s}$, or $\tilde{\mathbf{u}}, \mathbf{v} \prec \mathbf{s}$. Let us first assume that $\mathbf{u}, \mathbf{v} \prec \mathbf{s}$. Let $\mathbf{u}=u_{1} u_{2} \ldots u_{k}$ and $\mathbf{v}=v_{1} v_{2} \ldots v_{m}$, w.l.o.g. $k \leq m$. Finding $\mathbf{w}$ consists of interleaving $\mathbf{u}$ and $\mathbf{v}$ in such a way that the resulting string is a subsequence of $\mathbf{s}$. In other words, we must find indices $0 \leq i_{1} \leq i_{2} \leq \ldots \leq i_{k+1} \leq k+1$ s.t. $\mathbf{w}=$ $v_{1} \ldots v_{i_{1}} u_{1} v_{i_{1}+1} \ldots v_{i_{2}} u_{2} \ldots u_{k} v_{i_{k}+1} \ldots v_{i_{k+1}}$ is a subsequence of $\mathbf{s}$. (For the sake of conciseness, we set $v_{i}$ to be the empty string for $i<1$ and $i>m$.) This can be done by asking queries $v_{1} \mathbf{u}, v_{1} v_{2} \mathbf{u}, v_{1} v_{2} v_{3} \mathbf{u}$ etc. until the first no to determine $i_{1}$; then queries $v_{1} \ldots v_{i_{1}} u_{1} v_{i_{1}+1} u_{2} u_{3} \ldots u_{k}, v_{1} \ldots v_{i_{1}} u_{1} v_{i_{1}+1} v_{i_{1}+2} u_{2} u_{3} \ldots u_{k}$, etc. to determine $i_{2}$. Proceeding in this way, we will find all $i_{j}$ 's, using $i_{j}-i_{j-1}+1$ many queries in the $j$ 'th step, so altogether $i_{1}+1+\sum_{j=2}^{k+1}\left(i_{j}-i_{j-1}+1\right)=$ $m+k+1=|\mathbf{v}|+|\mathbf{u}|+1$ many queries.

Now note that we have assumed that both $\mathbf{u}$ and $\mathbf{v}$ are subsequences of $\mathbf{s}$. In this case, the string $\mathbf{w}$ which is constructed is also a subsequence of $\mathbf{s}$. If, on the other hand, both $\mathbf{u}$ and $\mathbf{v}$ are subsequences of $\tilde{\mathbf{s}}$, then the $\mathbf{w}$ thus constructed will also be a subsequence of $\tilde{\mathbf{s}}$, thus satisfying $\mathbf{w} \prec_{\mathrm{RC}} \mathbf{s}$. Finally, if neither of these cases holds, then the algorithm sketched above will abort without producing a desired $\mathbf{w}$. Then we repeat it with $\mathbf{u}$ and $\tilde{\mathbf{v}}$, which will produce a string $\mathbf{w}$ that is either a subsequence of $\mathbf{s}$ or of $\tilde{\mathbf{s}}$, in either case $\mathbf{w} \prec_{\mathrm{RC}} \mathbf{s}$, as claimed. The total number of queries is thus at most $2(|\mathbf{u}|+|\mathbf{v}|+1)$.

Theorem 3 There exists an algorithm for reconstructing a string using $\Theta(n \log |\Sigma|)$ $R C$-subsequence queries of unbounded length. 
Proof. We first give the algorithm for the case where $|\Sigma|=2$. Let $\Sigma=\{a, b\}$ where $b=\bar{a}$. Let $M=\max \left\{j \geq 0 \mid a^{\chi} \prec_{\mathrm{RC}} \mathbf{s}\right\}$. Clearly, we have $M=$ $\max _{c=a, b}|\mathbf{s}|_{c}$. Notice that we can determine $M$ by asking query $\mathbf{t}_{\chi}^{(0)}=a^{\chi}$, for $\chi=\left\lceil\frac{n}{2}\right\rceil,\left\lceil\frac{n}{2}\right\rceil+1, \ldots$, until we get the first negative answer, implying that $M=\chi-1$. In particular, we need exactly $M+1$ such queries.

Define $y_{1}=\max \left\{\chi=0,1,2, \ldots, \mid b^{\chi} a^{M} \prec_{\mathrm{RC}} \mathbf{s}\right\}$, and for $i=2, \ldots, M+1$,

$$
y_{i}=\max \left\{\chi=0,1,2, \ldots \mid b^{y_{1}} a b^{y_{2}} a \ldots b^{y_{i-1}} a b^{\chi} a^{M-i+1} \prec_{\mathrm{RC}} \mathbf{s}\right\} .
$$

In perfect analogy with what we did above, we can determine $y_{i}$, by asking the query $\mathbf{t}_{\chi}^{(i)}=b^{y_{1}} a b^{y_{2}} a \ldots b^{y_{i-1}} a b^{\chi} a^{M-i+1}$, for each $\chi=1,2, \ldots$, until we receive a negative answer, implying that $y_{i}=\chi-1$. Therefore, we need exactly $y_{i}+1$ such queries to determine $y_{i}$.

Let us write $\mathbf{t}$ for $\mathbf{t}_{y_{M+1}}^{(M+1)}$. For constructing $\mathbf{t}$ we need exactly $M+1+$ $\sum_{i=1}^{M+1}\left(y_{i}+1\right)=2 \max _{c=a, b}|\mathbf{s}|_{c}+2+\min _{c=a, b}|\mathbf{s}|_{c}$ many queries. We claim that $\mathbf{t}$ is a maximum size subsequence of $\mathbf{s}$ or $\mathbf{s}^{\prime}$, i.e., there exists no $\mathbf{t}^{\prime}$ such that $\mathbf{t} \prec \mathbf{t}^{\prime} \prec_{\mathrm{RC}} \mathbf{s}$. This implies that $\mathbf{t} \in\{\mathbf{s}, \tilde{\mathbf{s}}\}$, i.e., the above procedure determines $\mathbf{s}$ up to reverse complement using $O(n)$ queries.

We prove the claim by contradiction. Assume that $\mathbf{t} \notin\{\mathbf{s}, \tilde{\mathbf{s}}\}$. Since by construction $\mathbf{t} \prec_{\mathrm{RC}} \mathbf{s}$, it follows that there exists a sequence $\mathbf{t}^{\prime} \neq \mathbf{t}$ such that $\mathbf{t} \prec \mathbf{t}^{\prime} \prec_{\mathrm{RC}} \mathbf{s}$, and $\left|\mathbf{t}^{\prime}\right|=|\mathbf{t}|+1$.

By the definition of $M$, it follows that $|\mathbf{t}|_{a}=\left|\mathbf{t}^{\prime}\right|_{a}$, for otherwise $a^{M+1} \prec$ $\mathbf{t}^{\prime} \prec_{\mathrm{RC}} \mathbf{s}$, contradicting the fact that $M$ is maximal. Therefore it must be $\left|\mathbf{t}^{\prime}\right|_{b}=|\mathbf{t}|_{b}+1$. In analogy with what we have done for $\mathbf{t}$, let us write $\mathbf{t}^{\prime}=$ $b^{y_{1}^{\prime}} a b^{y_{2}^{\prime}} a \ldots b^{y_{M}^{\prime}} a b^{y_{M+1}^{\prime}}$, where $y_{j}^{\prime} \geq 0$, for each $j=1, \ldots, M+1$. By the definition of $\mathbf{t}^{\prime}$, there exists exactly one $j$ such that $y_{j}^{\prime}=y_{j}+1$. It follows that $b^{y_{1}} a b^{y_{2}} a \ldots b^{y_{j-1}} a b^{y_{j}+1} a^{M-j} \prec \mathbf{t}^{\prime} \prec_{\mathrm{RC}} \mathbf{s}$, which contradicts the definition of $y_{j}$.

Now let $|\Sigma|=2 \delta$, with $\delta>1$. For each $i=1, \ldots, \delta$, let us denote by $\mathbf{s}_{\mid i}$ the longest subsequence of $\mathbf{s}$ only containing characters from $\left\{a_{i}, \overline{a_{i}}\right\}$. We call $\mathbf{s}_{\mid i}$ the $i$ 'th projection of $\mathbf{s}$.

It is not hard to see that we can use the above procedure to identify a string $\mathbf{u}_{i} \in\left\{\mathbf{s}_{\mid i}, \tilde{\mathbf{s}}_{\mid i}\right\}$. In particular, it follows that the number of queries required for determining the $i$ 'th projection is thus at most $\frac{3}{2} A_{i}+2$, where $A_{i}=\left|\mathbf{s}_{\mid i}\right|$.

Once we have identified the $i$ th projection of $\mathbf{s}$ (up to reverse complement), for each $i=1, \ldots, \delta$, we can use Lemma 12 for iteratively interleaving these projections and constructing $\mathbf{s}$ : We first interleave $\mathbf{s}_{\mid 1}$ with $\mathbf{s}_{\mid 2}$, which yields $\mathbf{s}_{\mid 1,2}$. Then we interleave $\mathbf{s}_{\mid 1,2}$ with $\mathbf{s}_{\mid 3,4}$ and so on. By Lemma 12, each of these can be done using at most twice the total length of the two strings plus 2. So the total number of queries for the interleaving phase is at most $2 n \log \delta+2(\delta-1)$, since the lengths of the subsequences at each level add up to $n$, there are $\log \delta$ many levels, and for each of the $\delta-1$ many inner nodes, where the interleavings happen, we may have two additional queries. Thus the total number of queries for the complete algorithm is $\sum_{i=1}^{\delta}\left(\frac{3}{2} A_{i}+2\right)+2 n \log \delta+2(\delta-1)=\frac{3}{2} n+2 n \log \delta+4 \delta-2=$ $O(n \log |\Sigma|)$, using the fact that $|\Sigma|=2 \delta$ and the (natural) assumption that $|\Sigma|=O(n)$. 


\section{Missing proofs of Section 4.4}

Lemma 10. Fix $i<\rho$ and assume that for each $k=1, \ldots, i-1$, we know $r_{k}$ and $r_{2 \rho-k+1}$ and it holds that $r_{k}=r_{2 \rho-k+1}<T-\frac{n}{2}$. Then we can determine $m_{i}$ and $\min \left\{M_{i}, T-\frac{n}{2}\right\}$, asking at most $\max \left\{m_{i}, \min \left\{M_{i}, T-\frac{n}{2}\right\}\right\}$ queries.

Proof. For each odd $i$ (i.e., $r_{i}$ denotes the length of a run of $a$ 's) we have

$$
\begin{gathered}
m_{i}=\min \left\{\chi=1,2,3, \ldots \mid \mathbf{t}_{\chi}=a^{x_{1}+\cdots+x_{i-1}+\chi} b a^{\frac{n}{2}-\left(x_{1}+\cdots+x_{i-1}+\chi\right)} \prec_{\mathrm{RC}} \mathbf{s}\right\}, \\
\min \left\{M_{i}, T-\frac{n}{2}\right\}=\max \left\{\chi=m_{i}, m_{i}+1, \ldots, T-\frac{n}{2} \mid\right. \\
\left.\mathbf{q}_{\chi}=a^{\frac{n}{2}-\left(y_{1}+\cdots+y_{i-1}\right)} b^{\chi} a^{y_{1}+\cdots+y_{i-1}} \prec_{\mathrm{RC}} \mathbf{s}\right\} .
\end{gathered}
$$

Using (8), one can determine the value $m_{i}$ (resp. $M_{i}$ ) by asking the query $\mathbf{t}_{\chi}$ (resp. $\mathbf{q}_{\chi}$ ) for increasing values of $\chi$, until the first positive (resp. negative) answer. This settles the case of $i$ odd.

It is not hard to see that exactly the same argument holds for even $i$, using the following:

$$
\begin{aligned}
m_{i} & =\min \left\{\chi=1,2, \ldots \mid \mathbf{t}_{\chi}=b^{y_{1}+\cdots+y_{i-1}+\chi} a b^{\frac{n}{2}-\left(y_{1}+\cdots+y_{i-1}+\chi\right)} \prec_{\mathrm{RC}} \mathbf{s}\right\} \\
\min \left\{M_{i}, T-\frac{n}{2}\right\}=\max \{\chi & =m_{i}, m_{i}+1, \ldots, T-\frac{n}{2} \mid \\
\mathbf{q}_{\chi} & \left.=b^{\frac{n}{2}-\left(x_{1}+\cdots+x_{i-1}\right)} a^{\chi} b^{x_{1}+\cdots+x_{i-1}} \prec_{\mathrm{RC}} \mathbf{s}\right\} .
\end{aligned}
$$

This completes the proof of the lemma.

Lemma 11. Let $\mathbf{t}$ be defined as in Eq. (5). Then, it holds that

1. $\mathbf{t} \prec_{\mathrm{RC}} \mathbf{s}$ if and only if $\mathbf{t}^{\prime} \prec_{\mathrm{RC}} \mathbf{s}^{\prime}$.

2. If $\left|\mathbf{t}^{\prime}\right| \leq \frac{2\left(n^{\prime}+1\right)}{3}$, then $|\mathbf{t}| \leq \frac{2(n+1)}{3}$.

Proof. 1. Let $\mathbf{t} \prec_{\mathrm{RC}} \mathbf{s}$. First assume that $\mathbf{t} \prec \mathbf{s}$. Notice that $\mathbf{t}_{+}^{\prime}$ starts with a $b$ and ends with an $a$, and that $\mathbf{t}=a^{|\mathbf{u}|_{a}} \mathbf{t}_{+}^{\prime} a^{|\tilde{\mathbf{u}}|_{a}}$, i.e., the number of $a$ 's in $\mathbf{t}$ following $\mathbf{t}_{+}^{\prime}$ equals the number of $a$ 's in $\tilde{\mathbf{u}}$. Because of the $|\mathbf{u}|_{a}$ many $a$ 's at the beginning of $\mathbf{t}$, the fact that $\mathbf{t}$ is a subsequence of $\mathbf{s}$ implies $\mathbf{t}_{+}^{\prime} a^{|\tilde{\mathbf{u}}|} \prec a^{x_{i}} \mathbf{s}^{\prime} b^{y_{\rho-i+1}} \tilde{\mathbf{u}}$, and because $\mathbf{t}_{+}^{\prime}$ starts with a $b$, we also have $\mathbf{t}_{+}^{\prime} a^{|\tilde{\mathbf{u}}|} \prec \mathbf{s}^{\prime} b^{y_{\rho-i+1}} \tilde{\mathbf{u}}$. This again implies that $\mathbf{t}_{+}^{\prime} \prec \mathbf{s}^{\prime} b^{y_{\rho-i+1}}$, and because $\mathbf{t}_{+}^{\prime}$ ends with an $a$, also $\mathbf{t}_{+}^{\prime} \prec \mathbf{s}^{\prime}$, and thus, $\mathrm{t} \prec \mathbf{s}^{\prime}$.

Now let $\mathbf{t} \prec \tilde{\mathbf{s}}$, or, equivalently, $\tilde{\mathbf{t}} \prec \mathbf{s}$. We have $\tilde{\mathbf{t}}=b^{|\mathbf{u}|_{b}} \tilde{\mathbf{t}}_{+}^{\prime} b^{|\mathbf{u}|_{a}}=b^{|\mathbf{u}|_{b}} \tilde{\mathbf{t}}_{+}^{\prime} b^{|\tilde{\mathbf{u}}|_{b}}$, and $\tilde{\mathbf{t}}_{+}^{\prime}$ starts with an $a$ and ends with a $b$. Thus, because of the $|\mathbf{u}|_{b}$ many $b$ 's at the beginning of $\tilde{\mathbf{t}}$ and the fact that $\tilde{\mathbf{t}}_{+}^{\prime}$ starts with an $a$, we have $\tilde{\mathbf{t}}_{+}^{\prime} \prec \mathbf{s}^{\prime} b^{y_{\rho-i+1}} \tilde{\mathbf{u}}$. Further, because of the $|\tilde{\mathbf{u}}|_{b}$ many $b$ 's at the end and the fact that $\tilde{\mathbf{t}}_{+}^{\prime}$ ends with a $b$, this implies $\tilde{\mathbf{t}}_{+}^{\prime} \prec \mathbf{s}^{\prime}$. It follows that $\mathbf{t}^{\prime} \prec \mathbf{s}^{\prime}$. 
Conversely, if $\mathbf{t}^{\prime}$ (resp. $\tilde{\mathbf{t}}^{\prime}$ ) is a subsequence of $\mathbf{s}^{\prime}$, then clearly, $\mathbf{t}$ (resp. $\tilde{\mathbf{t}}$ ) is a subsequence of $\mathbf{s}$.

2. The length of $\mathbf{t}$ is $|\mathbf{t}| \leq|\mathbf{u}|+2+\left|\mathbf{t}^{\prime}\right|$, where $\left|\mathbf{t}^{\prime}\right| \leq \frac{2}{3}\left(n^{\prime}+1\right)$ and $n^{\prime}=$ $n-2|\mathbf{u}|-x_{i}-y_{\rho-i+1}$, and $y_{\rho-i+1}>x_{i} \geq 1$. This implies $x_{i}+y_{\rho-i+1} \geq 3$. Thus,

$$
\begin{aligned}
|\mathbf{t}| & \leq|\mathbf{u}|+2+\frac{2}{3}\left(n-2|\mathbf{u}|-x_{i}-y_{\rho-i+1}+1\right) \\
& =\frac{2}{3}(n+1)+2-\frac{1}{3}|\mathbf{u}|-\frac{2}{3}\left(x_{i}+y_{\rho-i+1}\right) \\
& \leq \frac{2}{3}(n+1)+2-\frac{1}{3}|\mathbf{u}|-2 \leq \frac{2}{3}(n+1) .
\end{aligned}
$$

